\title{
Ten-Year Evaluation of Cementless Buechel-Pappas Meniscal Bearing Total Ankle Replacement
}

\author{
Frederick F. Buechel, Sr., M.D.*; Frederick F. Buechel, Jr., M.D. ${ }^{\dagger}$; Michael J. Pappas, Ph.D. ${ }^{\ddagger}$
}

Newark, NJ; Philadelphia, PA

\begin{abstract}
A porous-coated, cementless, congruent-contact, threepiece, meniscal-bearing total ankle replacement was developed and used clinically over a 2- to 10-year period for patients with disabling ankle arthritis. Polished titanium-nitride ceramic-coated $\mathrm{Ti}_{6} \mathrm{Al}_{4} \mathrm{~V}$ tibial and talar components with a deep-sulcus trochlear groove and two lateral fixation fins for the talar onlay component were used. The ultra-high-molecular-weight polyethylene (UHMWPe) meniscal bearing congruently conformed to the flat upper tibial component surface and the deep sulcus and cylindrical geometry of the lower talar component surface. Fifty deep-sulcus (Buechel-Pappas ${ }^{\mathrm{TM}}$ ) total ankle replacements were implanted in 49 patients. Diagnoses were 8 osteoarthritis (16\%), 7 rheumatoid arthritis (14\%), 2 avascular necrosis (4\%), and 33 post-traumatic arthritis $(66 \%)$. Ages ranged from 26 to 71 years (mean 49 years). Clinical results using a strict ankle scoring system demonstrated good/excellent results in $88 \%$ of cases. Postoperative ankle motion ranged from $12^{\circ}$ to $46^{\circ}$ total arc (mean $28^{\circ}$ ), which was similar to the preoperative motion. Revision for malalignment was necessary in two cases (4\%). Mechanical complications included one case of meniscal bearing wear $(2 \%)$ in a patient with posttraumatic arthritis with component malalignment and one case of talar component subsidence $(2 \%)$ in a patient with avascular necrosis of the talus. No tibial component loosening was seen. Cumulative survivorship using an end point of revision of any component for any reason was $93.5 \%$ at 10 years (confidence interval $61-100 \%$ ).
\end{abstract}

*Department of Orthopaedic Surgery, UMDNJ-New Jersey Medical School, Newark, NJ

†Pennsylvania Hospital, University of Pennsylvania, Adult Reconstruction Fellow, Philadelphia, PA

‡Department of Mechanical Engineering, New Jersey Institute of Technology, Newark, NJ

Corresponding Author:

Frederick F. Buechel, M.D.

South Mountain Orthopedic Associates

61 First Street

South Orange, NJ 07079

E-mail: fbuechelmd@hotmail.com

For information on prices and availability of reprints call 410-494-4994 X226
Key Words: Ankle Replacement; Cementless Fixation; Ankle Arthritis; Mobile Bearing Ankle Replacement

\section{INTRODUCTION}

Loads in the ankle are similar to those in the knee, ${ }^{23}$ and yet the ankle is much smaller, making excessive contact stress a particular problem in ankle replacement. ${ }^{5}$ Fixed-bearing knees are typically strained well beyond the reasonable allowable stress ${ }^{6}$; thus the use of a congruent mobile bearing in ankle replacement may be more important than it is in knee replacement.

Inversion-eversion congruity is even more essential in ankle joint replacement to prevent edge-loading wear and deformation, which has been extensively reported with flat-on-flat knee replacement designs (Fig. 1). ${ }^{18}$ Additionally, deepening the trochlear sulcus angle tends to center the meniscal bearing and prevent bearing subluxation under normal load-bearing conditions. ${ }^{4}$ The improved stability of the deep-sulcus geometry (no subluxations) over the shallow-sulcus geometry $(10 \%$ subluxations) has been reported in the short term. ${ }^{4}$

Ankle replacement designs using mobile bearings have significantly improved the results of ankle arthroplasty in the last decade without the need for major bone resection or fusion of the distal tibiofibular syndesmosis. ${ }^{4,14}$ These results have been produced by allowing normal kinematic function of the bones and ligaments as seen in gait analysis ${ }^{19}$ and loadbearing cineradiographic studies. ${ }^{15}$ Such normal kinematic ankle function involves distal fibular rotation to optimize lateral ligamentous function during dorsi- and plantarflexion. Such rotation can average $2.2^{\circ}$ (range $\left.1.4^{\circ}-4.8^{\circ}\right){ }^{11}$ Fixed-bearing devices that seek to eliminate or minimize this fibular motion or restrict axial rotation can be subjected to torsional loads which can loosen tibial component fixation ${ }^{1,8,10,11,13,20,24,25}$ or result in nonunion of the syndesmosis. ${ }^{9,21}$

This study involves the use of rotationally unconstrained deep-sulcus meniscal bearings that 


\section{BI-SPHERICAL SURFACES}

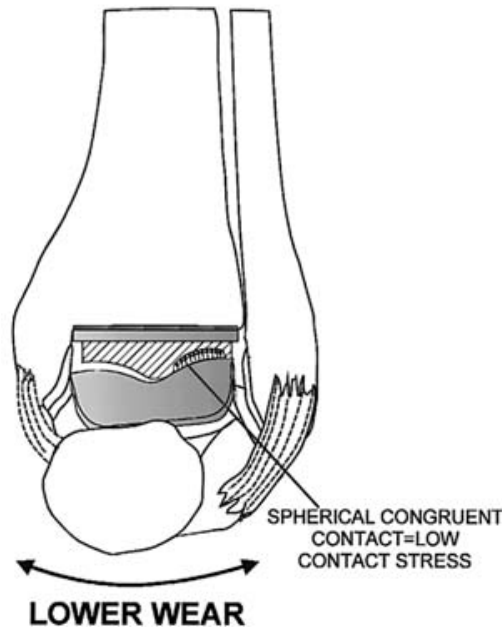

FLAT ON FLAT SURFACES

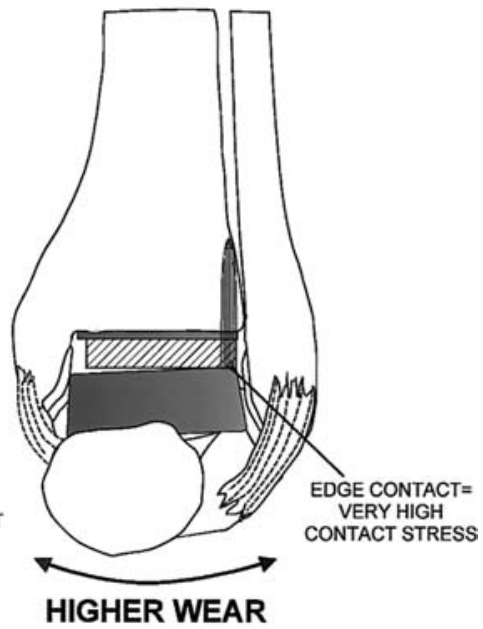

Fig. 1: Comparison of congruent deep-sulcus type and incongruent flat-on-flat type total ankle replacements during inversion-eversion.
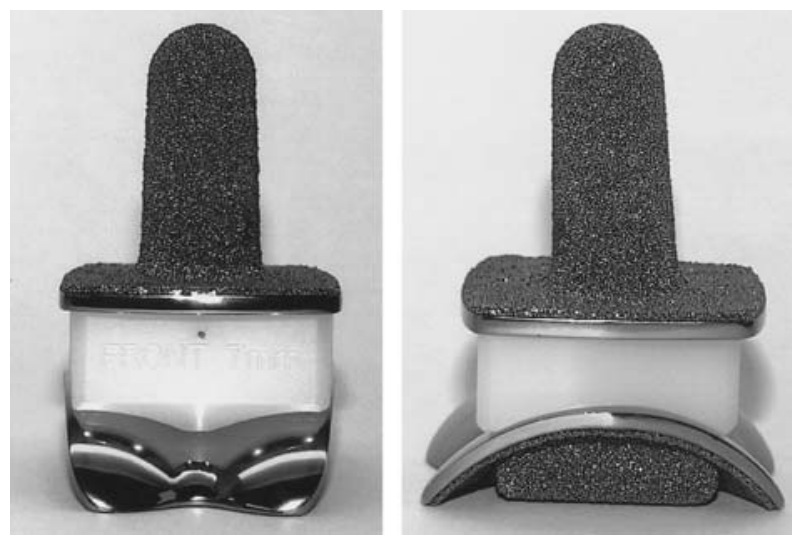

Fig. 2: Buechel-Pappas ${ }^{\mathrm{TM}}$ deep-sulcus, meniscal-bearing total ankle replacement.

articulate with a flat tibial plate superiorly and a biconcave trochlear talar component surface inferiorly (Fig. 2). The surface geometry is fully congruent, even during inversion and eversion motions to reduce contact stresses to $5 \mathrm{MPa}$, the medical load limit for ultra-highmolecular-weight polyethylene (UHMWPe). ${ }^{6}$

The current study reflects longer-term use of the deep-sulcus design with more detailed analysis of clinical, radiographic, and survivorship outcomes.

\section{MATERIALS AND METHODS}

A consecutive series of 50 cementless, porouscoated, congruent-contact, meniscal-bearing total ankle replacements (Buechel-Pappas ${ }^{\mathrm{TM}}$ Total Ankle, Endotec, South Orange, $\mathrm{NJ}$ ), implanted in 49 patients from January 1991 to December 1998 was prospectively evaluated using the New Jersey Orthopaedic Hospital $(\mathrm{NJOH})$ ankle evaluation scoring scale, ${ }^{5}$ most heavily weighted for pain (40 points) and function (40 points), with range of motion (15 points) and deformity (5 points) also recorded. This validated scoring scale ${ }^{16}$ is similar to the Mazur scale. ${ }^{17}$ Evaluations were performed at 3 months, 6 months, 1 year, and yearly thereafter by the same trained medical assistant, usually in person but occasionally by phone if a patient hardship was encountered. Ankle motion was measured clinically with a goniometer during office evaluations.

Kaplan-Meier ${ }^{12}$ survivorship analysis using an end point of revision of any component for any reason was performed to evaluate the long-term results. Radiographic analyses using coordinate axes of orientation were performed on satisfactory available $x$-rays in the early postoperative period and compared to the latest follow-up x-rays to determine component migration, wear, osteolysis, or bearing subluxation/dislocation.

\section{PATIENT DEMOGRAPHICS}

Diagnoses were 33 post-traumatic arthritis (PTA, $66 \%), 8$ osteoarthritis (OA,16\%), 7 rheumatoid arthritis (RA,14\%), and 2 avascular necrosis (AVN, 4\%). The breakdown of the post-traumatic patients was 2 talar fractures, 4 chronic lateral ligament sprains, 10 closed ankle fractures of undetermined cause, 6 fracture dislocations, 2 closed pilon fractures, 2 trimalleolar fractures, 5 bimalleolar fractures, 1 triple arthrodesis and 1 shotgun blast. The causes of avascular necrosis were prednisone for systemic lupus erythematosis and RA and IV steroids for a pulmonary problem of unknown origin. Ages ranged from 26 to 71 years (mean 49 years). 
Height ranged from $155 \mathrm{~cm}$ to $191 \mathrm{~cm}$ (mean $173 \mathrm{~cm}$ ). Weight ranged from $45 \mathrm{~kg}$ to $125 \mathrm{~kg}$ (mean $73 \mathrm{~kg}$ ). Preoperative ankle motion ranged from $0^{\circ}$ to $50^{\circ}$ total $\operatorname{arc}$ (mean $27^{\circ}$ total arc).

\section{OPERATIVE TECHNIQUE}

The patient was placed in the supine position with a sandbag beneath the buttock to roll the ankle into a neutral position.

A midline anterior straight, skin incision was centered on the ankle joint for a distance of approximately $15 \mathrm{~cm}$
(Fig. 3A). The superficial peroneal nerve branches were identified in the subcutaneous tissues, mobilized, and retracted lateralward and the ankle joint was exposed (Fig. 3, A-C). The anterior tibial osteophytes were removed to expose the superior surface of the talus. Using the tibial resection guide, the tibia was resected with a power saw at a $7^{\circ}$ posteriorly inclined angle, which was perpendicular to the tibial shaft axis in the A$P$ plane and approximately $10 \mathrm{~mm}$ from the upper talar surface (Fig. 4A). The medial malleolus was protected and all posterior bone fragments were morselized and removed. An anterior tibial window was marked with the window osteotome and a power saw was then used

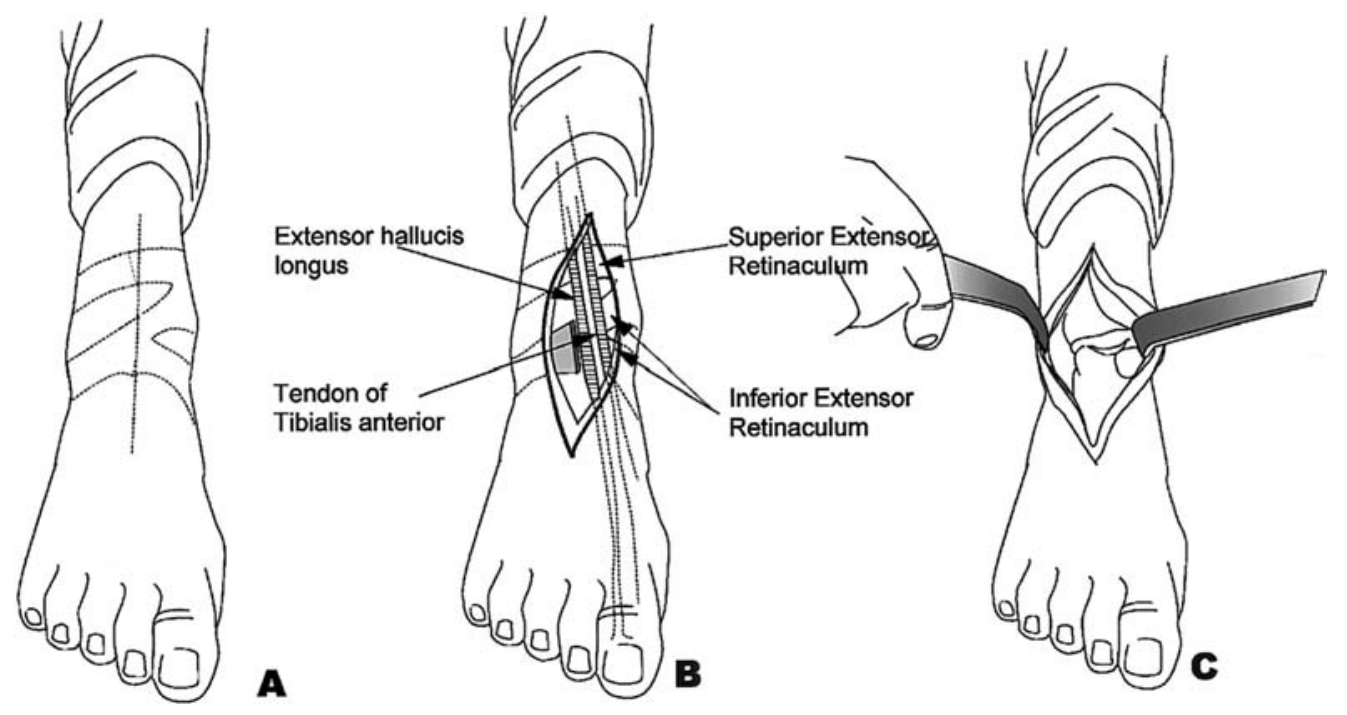

Fig. 3: A, Midline anterior straight skin incision over the ankle joint. B, Initial exposure. C, Deep exposure of the ankle joint.

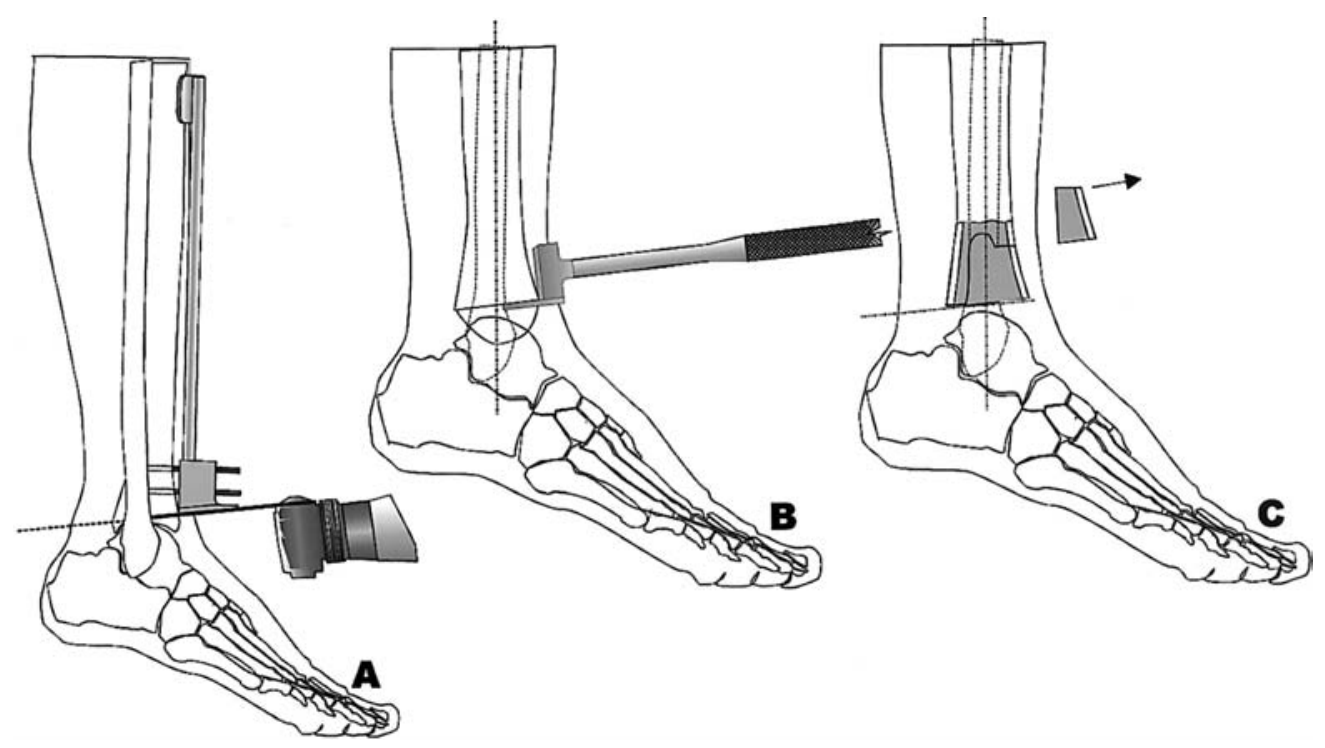

Fig. 4: A, Placement of tibial resection guide. B, An anterior tibial window is marked with the window osteotome. C, The bony window removed. 


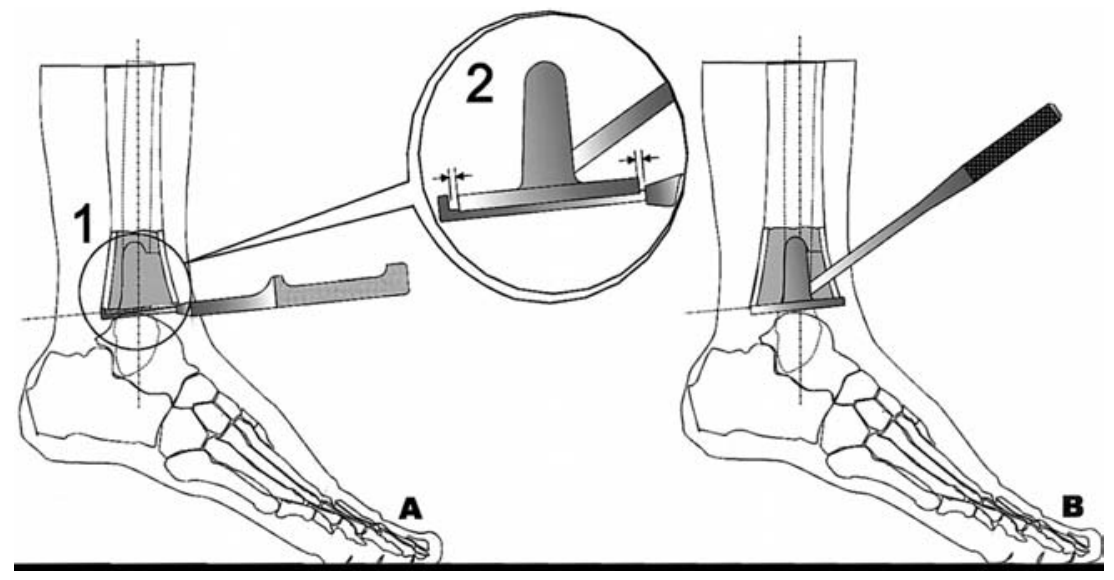

Fig. 5: A, Depth gauge used to center tibial component into the prepared tibial shaft. B, Tibial trial positioning.
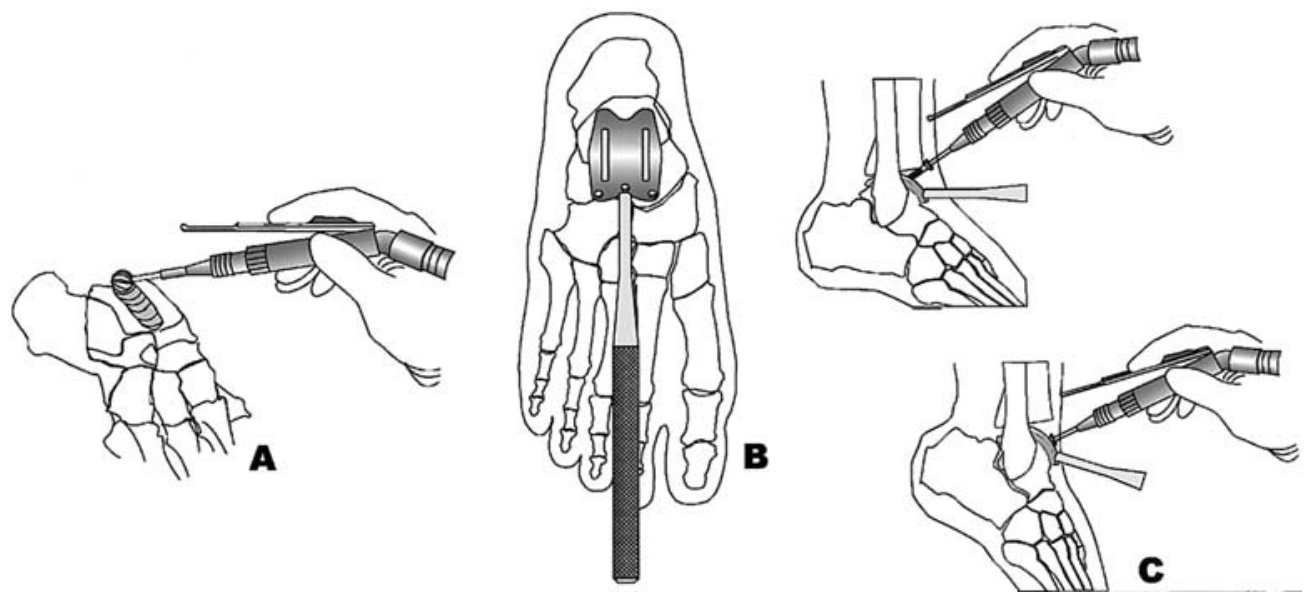

Fig. 6: A, Talus prepared with a 10-mm spherical burr to match the central sulcus. B, Talar template placement on talar component. C, The talar template slots are used to burr two fixation fin channels.

to cut the tibial window to a depth of approximately $2 \mathrm{~cm}$. The bony window was removed and placed in a blood-soaked sponge for later reimpaction (Fig. 4, B and C).

A depth gauge was used to measure the A-P diameter of the resected distal tibia, and the tibia and talus were prepared (Figs. 5 and 6). Once the tibial and talar preparations were completed, the trial tibial component was centered in the distal tibia followed by impaction of the trial talar component directly beneath the tibial component. Meniscal-bearing trials that match the talar component size were then pushed into place to established proper ligamentous tension (Fig. 7). A tight fit with no medial or lateral opening was preferred.

Dorsiflexion to at least neutral position was achieved. If not, a percutaneous three-puncture Achilles tendon lengthening was performed (one patient in this study). All trial components were removed and final components

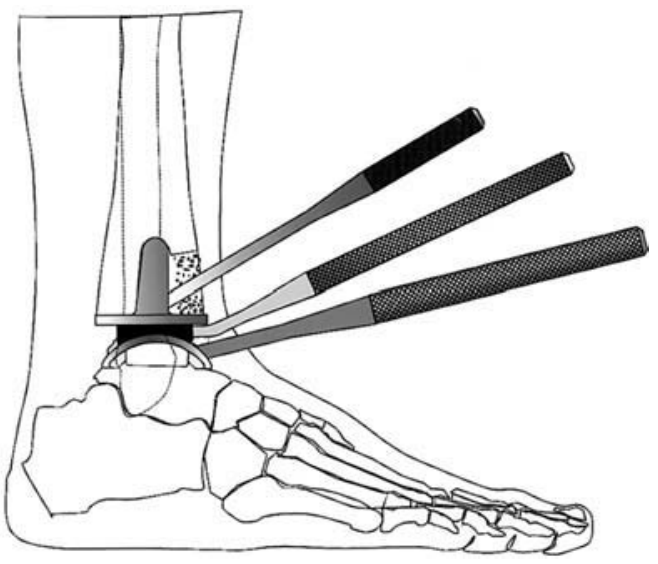

Fig. 7: Trial component implantation of tibia, talus, and bearing.

inserted as shown in Figure 8. The anterior cortical window graft was impacted into the distal tibia (Fig. 9). 


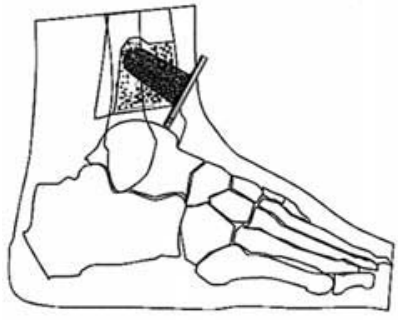

A

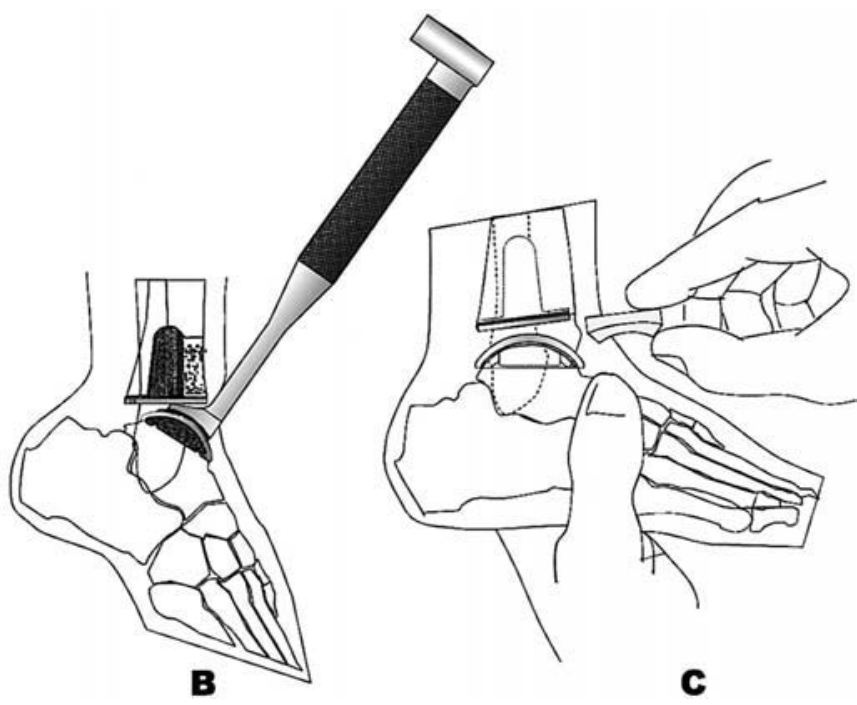

Fig. 8: A, Tibial component implantation. B, Talar component implantation. C, Insertion of meniscal bearing.

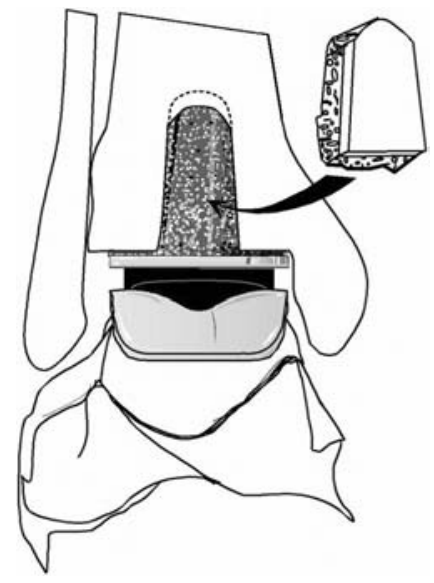

Fig. 9: Replacing the tibial window.

At the time of this study, subtalar and midfoot arthritis patients were not treated with any adjunctive procedures during total ankle replacement (TAR) surgery. Currently, it is felt that severe subtalar or midfoot arthritis should be managed by fusion, either before, during, or after TAR. Varus-valgus deformities need to be corrected prior to or during TAR to provide neutral hindfoot alignment. If lateral instability persists during TAR, then lateral ligament reconstruction needs to be performed.

After closure a short-leg plaster cast in neutral position of the foot and ankle was then applied.

\section{POSTOPERATIVE CARE}

Nasal oxygen (3-5 L/min) was administered continually for the initial 48 hours after surgery theoretically to improve initial wound healing. If extensive swelling occurred during the first 48 hours, the short-leg cast was bivalved laterally and medially. Weightbearing to tolerance was begun on the first postoperative day with intermittent elevation when nonambulatory. The initial plaster cast was removed and replaced with a fiberglass cast after 8 to 10 days. Final cast removal was performed at 6 weeks postoperatively, after which an ankle air-stirrup was applied for an additional 6 weeks to allow a return of dorsiflexion and plantarflexion motion. A high-top hiking-type shoe and support stockings were worn for 6 to 12 months until swelling had subsided and ankle strength had returned. Thereafter, comfortable footwear was worn during activities of daily living, which should include routine isometric and resistive dorsiflexion-plantarflexion and inversioneversion exercises to tolerance. Custom-molded shoes or corrective orthotic insoles may be prescribed for associated complex foot disorders.

\section{RESULTS}

Clinical evaluation using a strict ankle scoring scale ${ }^{5}$ demonstrated the overall results of cementless, Buechel-Pappas, meniscal-bearing total ankle replacements with 2 to 10 years follow-up (mean 5 years) to be 24 (48\%) excellent, 20 (40\%) good, 3 (6\%) fair, and 3 $(6 \%)$ poor. Postoperative ankle motion ranged from $12^{\circ}$ to $46^{\circ}$ total arc (mean $28^{\circ}$ total arc). Preoperative pain scores, which averaged 9.8 points (range 0-20 points) were improved postoperatively to an average 32.6 points (range 10-40 points). Thirteen out of 50 patients (26\%) described no pain postoperatively, 19 patients $(38 \%)$ had slight pain, 11 patients $(22 \%)$ had mild pain, 
3 patients $(6 \%)$ had moderate pain, and 4 patients $(8 \%)$ had severe pain. Pain was variable and most often related to soft tissue inflammatory conditions.

Moderate to severe postoperative pain caused limitation in daily activities for seven patients (14\%). Of the three patients with moderate pain, one patient had AVN of the talus, one post-traumatic arthritic patient with a triple arthrodesis developed impinging lateral bone spurs, and another post-traumatic arthritic patient with a lateral ligament reconstruction developed chronic posterior tibial tendinitis.

Of the four patients with severe pain, one patient had AVN of the talus, one post-traumatic arthritic patient developed a medial malleolar stress fracture that required revision, another post-traumatic arthritic patient developed reflex sympathetic dystrophy (RSD), and one osteoarthritic patient also developed RSD. The complications encountered in this study are summarized in Table 1 including those requiring revision. No fusions were necessary.

For the poor results, two of the three patients developed RSD symptoms, and the other patient developed medial and lateral stress fractures from varus malalignment. For the fair results, one was in an RA-lupus patient who developed talar component subsidence, another was in a lateral ligament instability patient that was reconstructed at TAR surgery, and the other in a patient who had a prior subtalar dislocation and continuing arthritis giving chronic subtalar pain. No definite correlations to weight or age can be made from this group.

Patient hardship in returning to the office for x-ray evaluation at the stated interval was frequently encountered. In addition, x-ray quality for all patients in the office setting was suboptimal for direct longitudinal comparison. Twenty-five patients with 26 ankle replacements had postoperative nonweightbearing $\mathrm{x}$-rays of satisfactory quality to evaluate radiolucencies around tibial and talar components. These were compared to preoperative $\mathrm{x}$-rays for overall alignment and followed for an average of 4.6 years (range $2-10$ years). Lucencies greater than or equal to $2 \mathrm{~mm}$ were seen to persist around two tibial components at 2 and 5 years, respectively. One tibial component was malpositioned anteriorly and was revised because of excessive bearing wear leading to osteolytic tibial cysts. Talar component lucencies greater than or equal to $2 \mathrm{~mm}$ were seen to persist around four talar components between 3 and 8 years postoperative. One of these four talar components also subsided in the bone approximately $3 \mathrm{~mm}$ based on sequential $\mathrm{x}$-rays and bony landmarks but no talar component revisions or bearing subluxations were encountered in this group. A typical successful Buechel-Pappas total ankle replacement x-ray after 8 years is seen in Figure 10.

Survivorship analysis ${ }^{12}$ using an end point of implant revision for any reason is shown in Figure 11. The Life Tables are shown in Table 2 for this population. The 10-year overall survival for the Buechel-Pappas design was $93.5 \%$.

\section{DISCUSSION}

There is evidence that mobile bearings have greatly improved the ability of surgeons to successfully replace multiaxial loading joints such as the ankle $\mathrm{e}^{4,11,13-15}$ while minimizing wear and loosening problems. Design improvements, such as deepening the talar sulcus geometry while maintaining bispherical congruity of the bearing surface have enhanced the long-term performance of mobile bearings for use in ankle joint replacement. 4,15 The improvements in implant stability using cementless technology, even under significant load bearing applications, are also noteworthy.

Observations made in this study support the design of mobile-bearing ankle replacements. Bearing subluxation problems previously encountered in a shallow-sulcus design ${ }^{4}$ were eliminated in the current deep-sulcus group by developing a sufficient sulcus

\begin{tabular}{|lccc}
\hline $\begin{array}{l}\text { Table 1: Complication of Buechel-Pappas } \\
\text { replacement }\end{array}$ & meniscal-bearing total ankle \\
Complications & \# of Ankles & $\%$ & Occurrence (years) \\
\hline Delayed wound healing & 7 & 14.0 & $0-0.3$ \\
Malleolar fracture & 3 & 6.0 & $0.2,0.8,2.7$ \\
Infection & 2 & 4.0 & $0.2,3.1$ \\
Reflex sympathetic dystrophy & 2 & 4.0 & $0.32,1.1$ \\
Talar component subsidence & 1 & 2.0 & 6.5 \\
Severe bearing wear & 1 & 2.0 & 4.7 \\
Bearing subluxation & 0 & 0.0 & - \\
Tibial component loosening & 0 & 0.0 & -
\end{tabular}



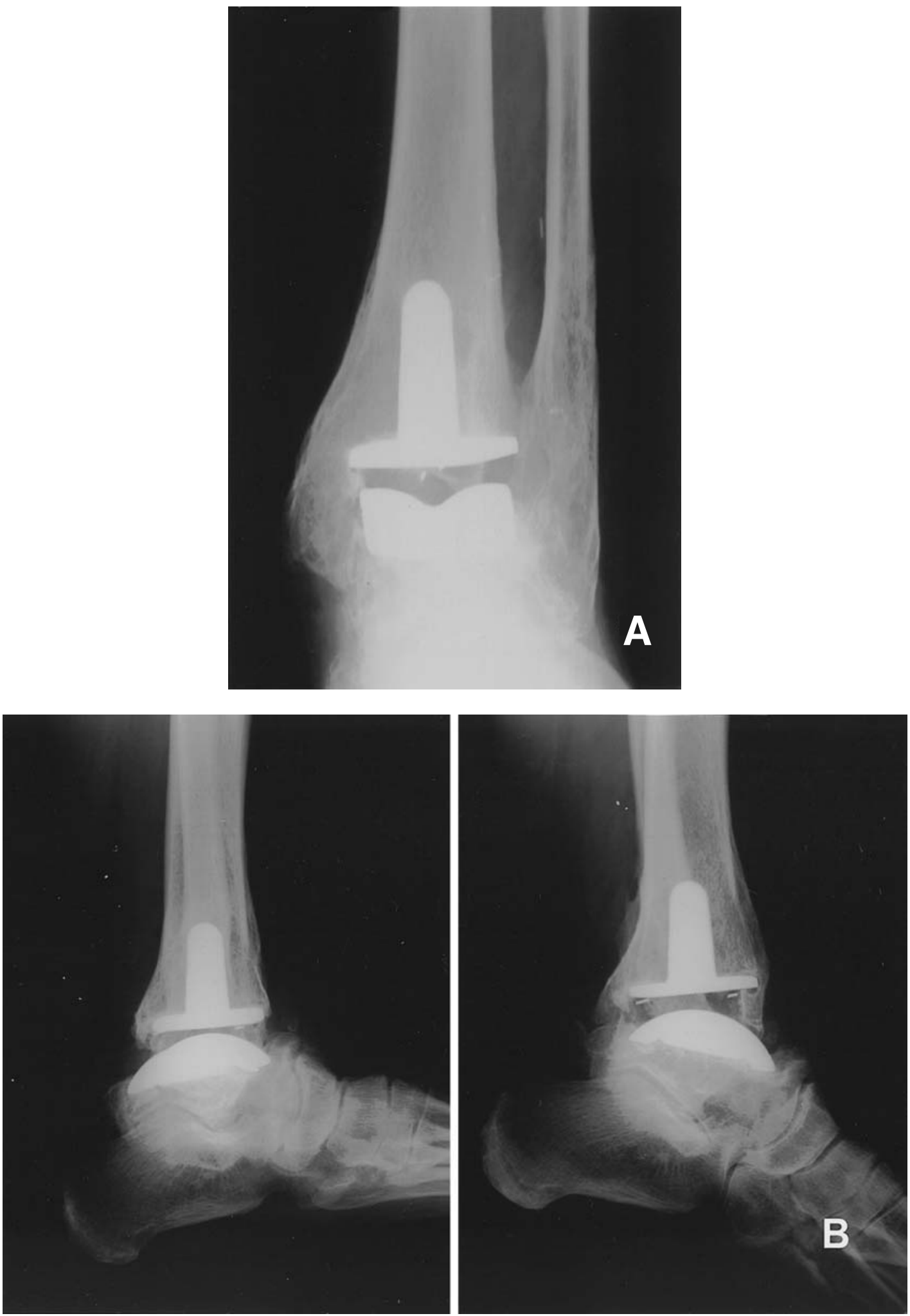

Fig. 10: A, Anteroposterior left ankle x-ray of a 61-year-old, 196-pound $(89 \mathrm{~kg}), 60$-inch $(152 \mathrm{~cm})$ tall, active woman, 8 years after deep-sulcus total ankle replacement for post-traumatic arthritis. B, Lateral dorsiflexion and plantarflexion $\mathrm{x}$-rays of the same patient at 8 years postoperative. 
BUECHEL-PAPPAS TOTAL ANKLE REPLACEMENT

SURVIVAL ESTIMATE

(ENDPOINT: REVISION FOR ANY REASON)

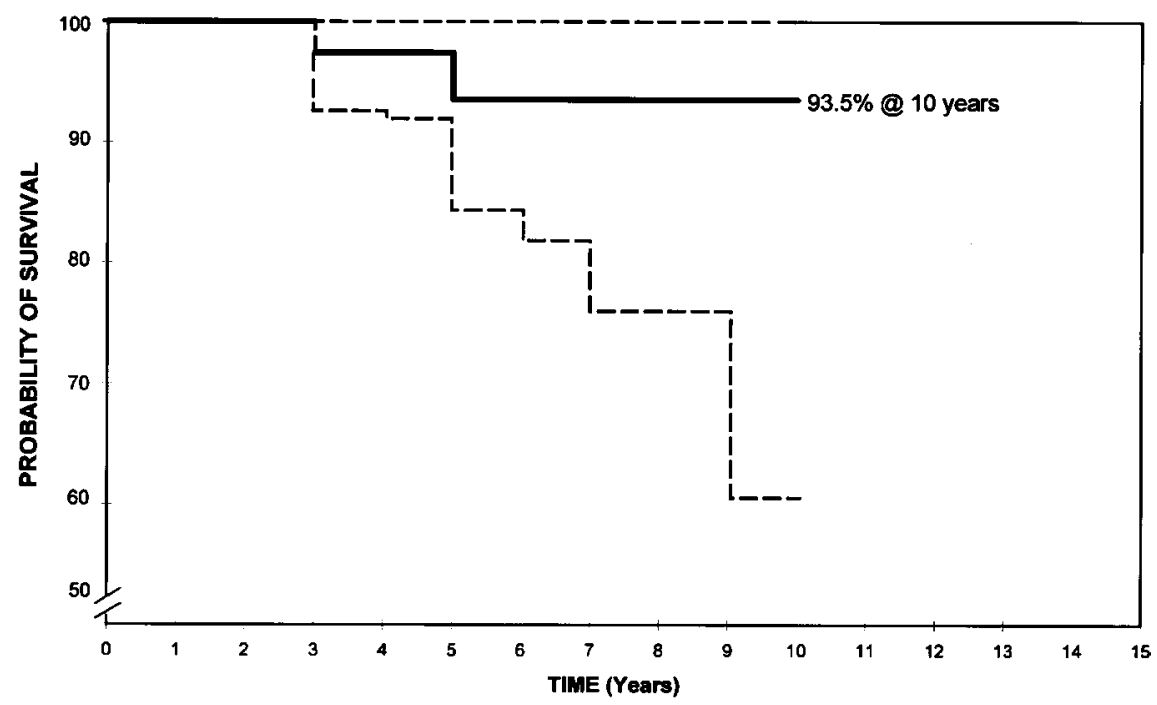

Fig. 11: Survivorship of cementless Buechel-Pappas ${ }^{\mathrm{TM}}$ meniscal-bearing total ankle replacement.

Table 2: Life tables for the Buechel-Pappas deep-sulcus total ankle replacement

\begin{tabular}{ccccccc}
$\begin{array}{l}\text { Follow-up } \\
\text { Interval }\end{array}$ & Failure & Censored & $\begin{array}{c}\text { Population } \\
\text { Size }\end{array}$ & Survival & ci- & ci+ \\
\hline 0 & 0 & 0 & 50 & 1.000 & - & - \\
1 & 0 & 1 & 49 & 1.000 & 1.00 & 1.00 \\
2 & 0 & 2 & 47 & 1.000 & 1.00 & 1.00 \\
3 & 1 & 8 & 39 & 0.974 & 0.93 & 1.00 \\
4 & 0 & 7 & 32 & 0.974 & 0.92 & 1.00 \\
5 & 1 & 7 & 25 & 0.935 & 0.84 & 1.00 \\
6 & 0 & 9 & 16 & 0.935 & 0.82 & 1.00 \\
7 & 0 & 9 & 7 & 0.935 & 0.76 & 1.00 \\
8 & 0 & 0 & 7 & 0.935 & 0.76 & 1.00 \\
9 & 0 & 5 & 2 & 0.935 & 0.61 & 1.00 \\
10 & 0 & 0 & 2 & 0.935 & 0.61 & 1.00 \\
\hline
\end{tabular}

angle to resist talar tilting and lateral displacement forces on the bearing. Such forces when applied in shallow-sulcus or flat articulations can cause medial or lateral bearing displacement, which destabilizes the replacement and leads to accelerated wear. Additionally, the volume of bearing thickness increases with a deeper-sulcus engagement, thus improving the tensile strength of the bearing to resist fracture. On this point, only one deep-sulcus bearing developed significant wear without a fracture (2\%), whereas 4 of 40 shallowsulcus meniscal bearings (10\%) developed wear-related fractures in a concurrent study. ${ }^{3}$

Wear in this study was seen in one patient $(2 \%)$ with malalignment of components in the lateral plane. ${ }^{2}$ The tibial component was anterior to the talar component causing the edge of the meniscal bearing to be unsupported on a flat edge surface posteriorly. This edge loading caused premature polyethylene bearing wear leading to two osteolytic cysts in the tibia (Fig. 12). Revision of the tibial component to a more posterior position corrected the problem (Fig. 13). Central positioning of the tibial and talar component is recommended to avoid this complication associated with edge loading.

Another design-related issue involves the fixation difference between a single central talar fixation fin and two off-center talar fixation fins. In a previous single-fin group (shallow-sulcus), six talar components 

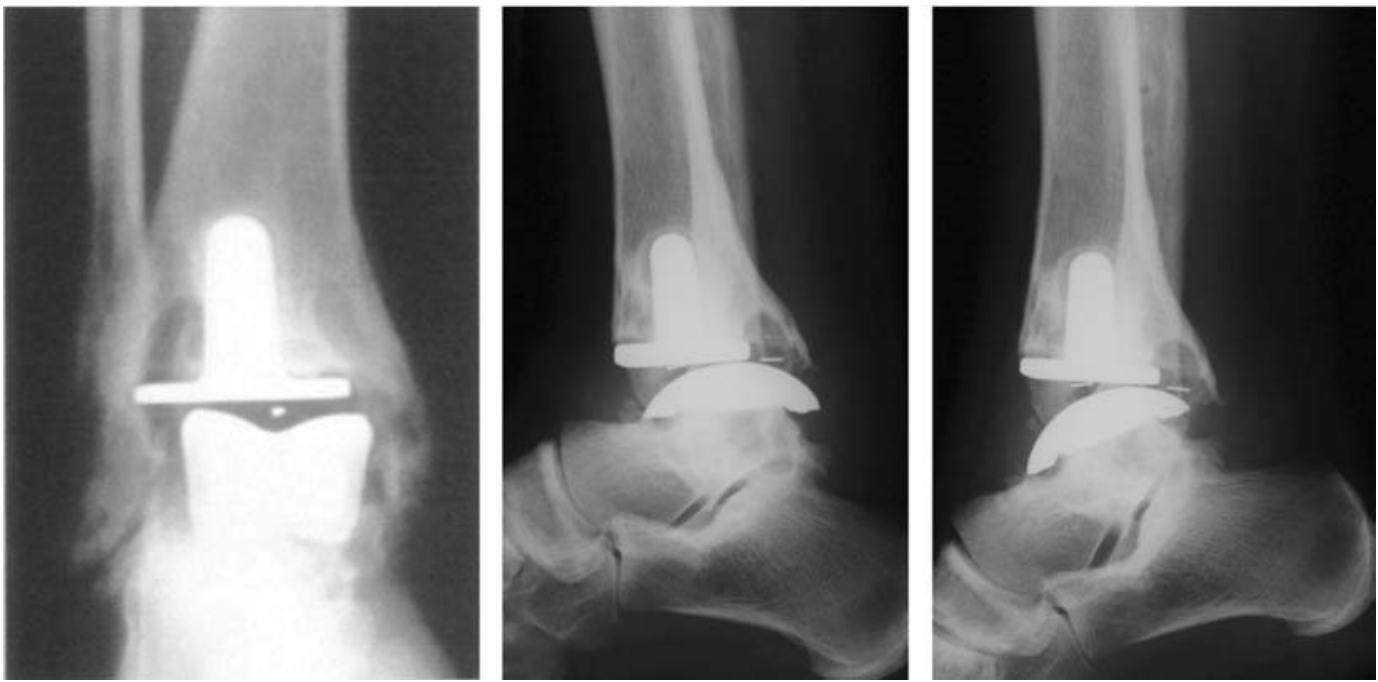

Fig. 12: Radiographs showing incorrect component placement, and subsequent premature polyethylene bearing wear leading to two osteolytic cysts in the tibia.
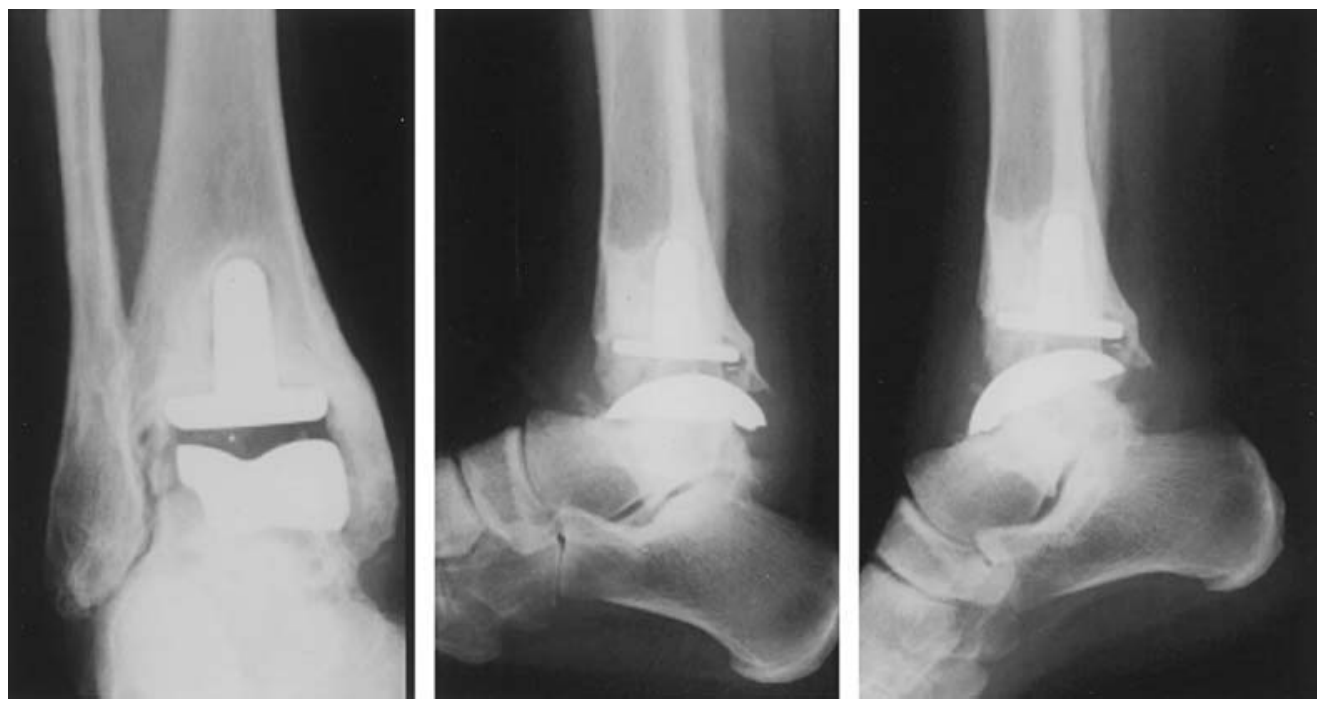

Fig. 13: Revision of the tibial component to a more posterior position to correct the problem seen in Figure 12.

(15\%) subsided, ${ }^{3}$ while in the current double-fin group (deep-sulcus), only one talar component (2\%) subsided. This improvement in talar component fixation and bearing stability strongly favors the deep-sulcus talar component design with two fixation fins.

The porous-coated tibial component loading stem and platform worked equally well in both designs with no gross migration or loosening observed. An interim tibial component with only $50 \%$ distal porous coating on the fixation stem showed ingrowth into the porous coating but a radiosclerotic "balloon-line" around the uncoated portion of the stem in 12 ankle replacements followed for 10 years (Fig. 14). Although none of these partially porous-coated tibial components loosened clinically, one component sustained a tibial plate fracture in an active osteoarthritic male patient. The radiographic pattern of ingrowth was superior in the fully porous-coated components seen in the current study. Additionally, the tibial component loading plate was strengthened to avoid further fatigue failures.

The absence of talar component side flanges on this deep-sulcus component did not appear to affect the quality of the clinical outcome over time. Although a theoretical concern over intermalleolar bone bridging was entertained, this phenomenon was rarely 

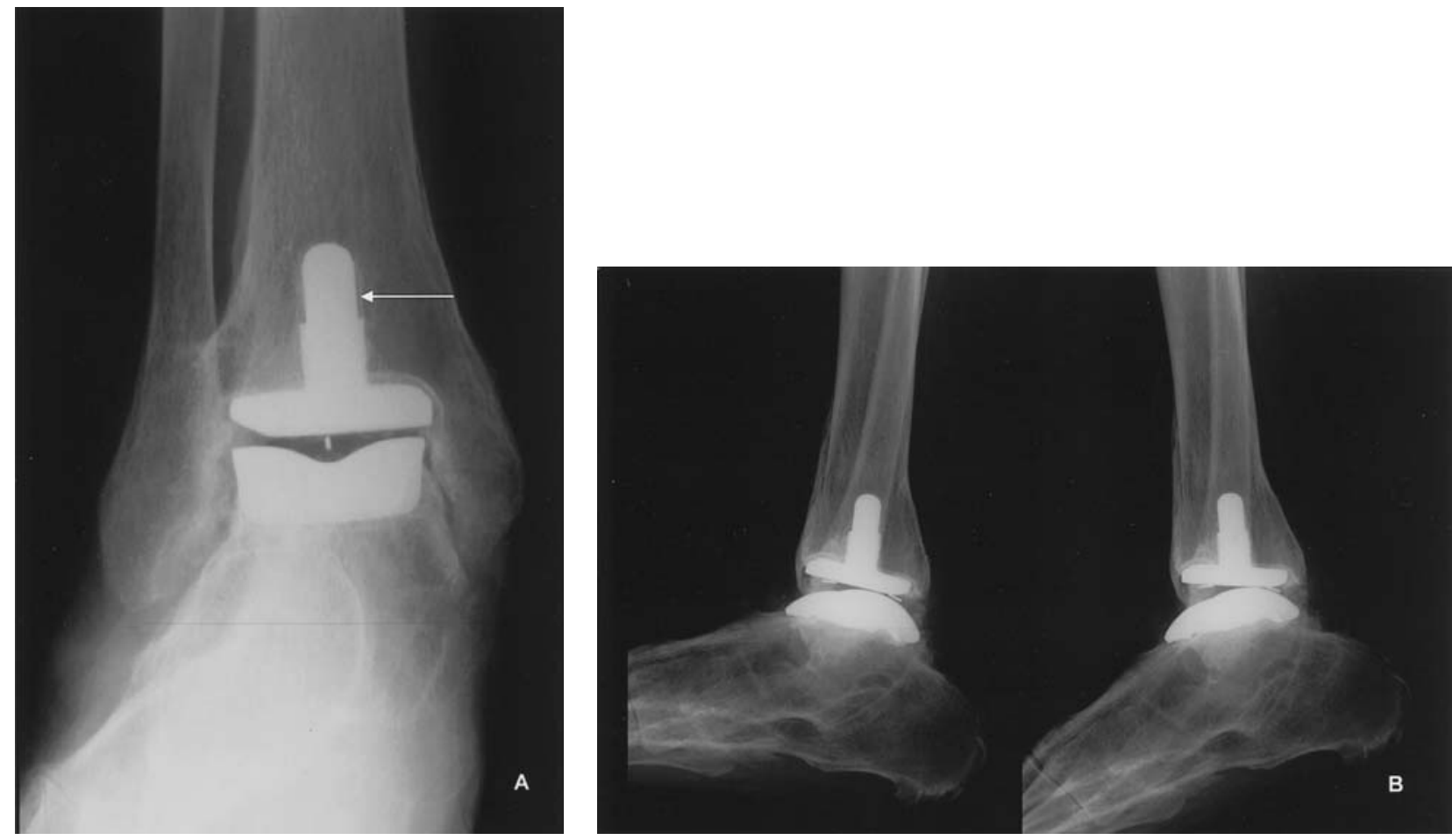

Fig. 14: A, Anteroposterior right ankle x-ray of a 64-year-old, 135-pound $(61 \mathrm{~kg}), 62$-inch $(158 \mathrm{~cm})$ tall, active woman with rheumatoid arthritis and spontaneous hindfoot fusion 10 years after deep-sulcus total ankle replacement using an interim tibial component with only $50 \%$ distal porous coating. The arrow points to a radiosclerotic "balloon-line" around the uncoated portion of the stem. B, Lateral dorsiflexion and plantarflexion $\mathrm{x}$-rays of the same patient at 10 years postoperative.

encountered, and postoperative range of motion $\left(28^{\circ}\right)$ remained similar to preoperative motion $\left(27^{\circ}\right)$, and did not improve. A more pressing concern is the possibility of stress shielding of the talus by using such covering flanges which eliminate malleolar bony contact, thus reducing normal malleolar loading patterns and introducing metal-on-bone articulations. Maintaining malleolar contact with the talus and allowing normal fibular rotation is desirable and appears to be a theoretical advantage of this design.

Of some additional concern is the x-ray observation of tibial bony overgrowth, anteriorly and posteriorly, around the tibial component loading plate over time even though no change in range of motion was observed in this study. The anteroposterior dimensions of the loading plate fall short of the true anteroposterior dimensions of the resected distal tibia, thus allowing osteogenesis and spur formation to form bony overgrowth which can limit range of motion. While, it is true that these spurs can be resected at a later date, it would be useful to prevent their formation. Such could be accomplished by extrapolating the total hip replacement data on use of indomethacin in the immediate postoperative period ${ }^{7}$ or by extending the anterior and posterior dimensions of the loading plate to help prevent the bony overgrowth. Both considerations are reasonable and are currently being implemented to improve ankle motion in the long term.

Delayed wound healing continues to be the most common complication (14\%) and perhaps is inevitable in this region of anatomy. Persistent diligence in minimizing this complication by extending the incisions and avoiding constant retraction pressure is recommended. In multiply operated ankles, incision planning to avoid wound compromise remains paramount.

Fracture of the medial malleolus (6\%) can be an annoying but not catastrophic problem, unless excessive varus of the ankle is involved which can lead to progressively increasing varus and later stress fracture of the medial malleolus. ${ }^{22}$ Such conditions may require realignment osteotomy to allow compressive rather than shearing loads at the ankle joint. Diligence to avoid varus malalignment of the ankle is recommended.

Infections following ankle joint replacement (4\%) remain worrisome, although antibiotic suppression was successful in both cases seen in this study. It is likely that the decreased vascularity in the ankle, especially after multiple surgical interventions, not only is responsible for significant delayed wound healing but also contributes to the overall increased incidence of 
infection seen. Patients with multiple surgical incisions or immunocompromised tissues should be alerted to these potential complications and should be prepared for prolonged wound care and possible plastic surgical intervention if needed.

RSD $(4 \%)$ remains a devastating complication following ankle joint replacement. Despite proper alignment of components and prolonged immobilization, this condition persists for many years and should be considered a lifetime condition with intermittent relapses. One patient in this study, encouragingly, improved after 5 years from a poor ankle score to good ankle score; the other RSD patient continues to have a poor score, despite excellent component alignment and fixation after 4 years.

The cementless Buechel-Pappas ${ }^{\mathrm{TM}}$ total ankle replacement, with its improvement in bearing stability, decreased talar component subsidence, and continued stable component fixation (survivorship $93.4 \%$ at 10 years, confidence interval 61-100\%) makes this prosthesis a useful alternative to ankle fusion in properly selected patients.

\section{REFERENCES}

1. Bolton-Maggs, BG; Sudlow, RA; Freeman, MAR: Total ankle arthroplasty: a long-term review of the London Hospital experience. J. Bone Joint Surg. 67-B:785-790, 1985.

2. Buechel, FF: Complications of total ankle replacement. Scientific Exhibit, Presented at American Academy of Orthopaedic Surgeons 68th Annual Meeting Specialty Day, San Francisco, CA, February 28 to March 4, 2001.

3. Buechel, Sr, FF; Buechel, Jr, FF; Pappas, MJ: Eighteenyear evaluation of cementless meniscal bearing total ankle replacements. In: JH Beaty, ed, Instructional Course Lectures, Vol. 51, Rosemont, IL, American Academy of Orthopaedic Surgeons, 2002, pp. 143-151.

4. Buechel, FF; Pappas, MJ: Survivorship and clinical evaluation of cementless, meniscal-bearing total ankle replacements. Semin. Arthrop. 3:43-50, 1992.

5. Buechel, FF; Pappas, MJ; lorio, L: New Jersey low contact stress total ankle replacement: biomechanical rationale and review of 23 cementless cases. Foot Ankle 8:279-290, 1988.

6. Buechel, FF; Pappas, MJ; Makris, G: Evaluation of contact stress in metal-backed patellar replacements: a predictor of survivorship. Clin. Orthop. 273:190-197, 1991.

7. D'Lima, DD; Venn-Watson, EJ; Tripuraneni, P; et al: Indomethacin versus radiation therapy for heterotopic ossification after hip arthroplasty. Orthopedics 24:1139-1143, 2001.
8. Groth, HE; Fitch, HF: Salvage procedures for complications of total ankle arthroplasty. Clin. Orthop. 224:245-249, 1987.

9. Hansen, ST: Cementless ankle arthroplasty in the United States of America: the Alvine agility total ankle arthroplasty. In: H Kofoed, ed, Current Status of Ankle Arthroplasty, New York, SpringerVerlag, 1998, pp. 37-40.

10. Hay, SM; Smith, TW: Total ankle arthroplasty: a long-term review. Foot 4:1-5, 1994.

11. Helweg, J; Kofoed, $\mathbf{H}$ : The fibula rotates during motion in the ankle joint. In: H Kofoed, ed, Current Status of Ankle Arthroplasty, New York, Springer-Verlag, 1998, pp. 59-63.

12. Kaplan, E; Meier, P: Nonparametric estimation from incomplete observations. J. Am. Statis. Assoc. 53:457-481, 1958.

13. Kofoed, H: Cylindrical cemented ankle arthroplasty: a prospective series with long-term follow-up. Foot Ankle 16:474-478, 1995.

14. Kofoed, H; Sorensen, T: Ankle arthroplasty for rheumatoid arthritis and osteoarthritis. Prospective long-term study of cemented replacements. J. Bone Joint Surg. 80-B:328-332, 1998.

15. Komistek, RD; Buechel, FF; Stiehl, JB; et al: A determination of ankle kinematics using fluoroscopy. Foot Ankle Int. 21:343-350, 2000.

16. Levi, N; Kofoed, $\mathbf{H}$ : Comparison of five different scoring systems of ankle arthroplasty. In: H Kofoed, ed, Current Status of Ankle Arthroplasty, New York, Springer-Verlag, 1998, pp. 135-137.

17. Mazur, JM; Schwartz, E; Simon, SR: Ankle arthrodesis: longterm follow-up with gait analysis. J. Bone Joint Surg. 61A:964-975, 1979.

18. Moran, CG; et al: Survivorship analysis of the uncemented porous coated anatomic knee replacement. J. Bone Joint Surg. 73-B:848-857, 1991.

19. Nelissen, RGHH; Doets, HC; Meskers, C: The value of ankle prostheses - A gait analysis approach. In: H Kofoed, ed, Current Status of Ankle Arthroplasty, New York, Springer-Verlag, 1998, pp. 72-77.

20. Pappas, MJ; Buechel, FF; DePalma, AF: Cylindrical ankle joint replacement. Clin. Orthop. 118:82-92, 1976.

21. Pyevich, MT; Saltzman, CL; Callaghan, JJ: Total ankle arthroplasty: a unique design. J. Bone Joint Surg. 80:1410-1420, 1998.

22. Saltzman, CL; Alvine, FG; Buechel, FF; et al: Complications of total ankle arthroplasty. Scientific Exhibit SE\#65, Presented at American Academy of Orthopaedic Surgeons, 68th Annual Meeting, San Francisco, CA, February 28 to March 4, 2001.

23. Stauffer, RN; Chao, E; Brewster, RC: Force and motion analysis of the normal, diseased and prosthetic ankle joint. Presented at the 23rd Annual Orthopaedic Research Society Convention, Las Vegas, NV, February 1-3, 1977.

24. Unger, AS; Inglis, AE; Mow, CS; Figgie, HE: Total ankle arthroplasty in rheumatoid arthritis: a long-term follow-up study. Foot Ankle 8:173-179, 1988

25. Wynn, AH; Wilde, AH: Long-term follow-up of conaxial (BechSteefee) total ankle arthroplasty. Foot Ankle 13:303-306, 1992. 